\title{
AN INEXPENSIVE AND CONTINUOUS RADON PROGENY DETECTOR FOR INDOOR AIR-QUALITY MONITORING
}

\author{
CHRISTIAN DI CARLO ${ }^{1,2}$, LUIGI LEPORE ${ }^{2}$, LUCA GUGLIERMETTI $^{2}$ \& ROMOLO REMETTI ${ }^{2}$ \\ ${ }^{1}$ Italian National Institute of Health, National Center for Radiation Protection and Computational Physics, Italy \\ ${ }^{2}$ Department of Basic and Applied Sciences for Engineering, Sapienza University of Rome, Italy
}

\begin{abstract}
A silicon photodiode-based inexpensive detector working as a counter and spectrometer for alpha particles has been conceived, designed, constructed and analyzed in depth. Monte Carlo simulations by means of MCNPX ver. 2.7.0 code have been carried out to select the most suitable sensitive element for the intended applications. The detecting unit has been coupled to an Arduino board and tested for low-rate alpha-particle counting and spectroscopy. Results demonstrate a maximum count rate of $4 \cdot 10^{3} \mathrm{~s}^{-1}$, an energy resolution corresponding to a full width at half maximum of $160 \mathrm{keV}$ over the entire energy range of measured alpha (namely $4 \div 6.5 \mathrm{MeV}$ ), and the sensitive element's intrinsic efficiency of about $100 \%$. Being the detector capable of distinguishing alpha energy associated to decays of radon daughters, its applications include ${ }^{222} \mathrm{Rn}$ progeny monitoring. The air sampling system has been realized by a volumetric micro-pump forcing the air-flow through a millipore filter. By knowing the air-flow rate processed and the corresponding alpha energy spectrum measured, the concentrations of ${ }^{218} \mathrm{Po}$, ${ }^{214} \mathrm{Po}$ and ${ }^{210} \mathrm{Po}$ are determined. The potential alpha energy concentration-in-air is inferred, and effective dose evaluated. Calibration and testing measurements have been carried out by comparing the obtained results to the outputs of professional and expensive radon progeny monitor. The detector capability of "following" radon progeny concentration-in-air vs. time has been demonstrated. The device studied here can be configured as a prototype for an inexpensive radon progeny sensor to be potentially suitable for indoor air-monitoring in residential buildings, evaluating people's exposures to radon and initiating corrective actions (e.g., mechanical ventilation) if necessary.
\end{abstract}

Keywords: radon exposure, alpha spectrometry, silicon photodiode, Arduino, PAEC.

\section{INTRODUCTION}

From 2013 on, the EU Member States have been busy in the transposition of Council Directive 2013/59/Euratom [1] into national legislation. Among novelties introduced, increased attention is paid to exposures from natural sources of radiation, and radon-related aspects particularly.

Updated primary risk model (BEIR VI [2] and Czech-French models [3]) studies regarding epidemiological approach to radon effects on health encouraged the review of the probability coefficient for radon- and radon-progeny-induced lung cancer from 2.8 E-4 to 5 E-4 per WLM [4]. The application of general biokinetic models (mainly the Human Respiratory Tract Model, HRTM, from ICRP 66 [5]) to radon exposures (including the progeny intakes in lungs and whole body) results in a values of effective dose per unit exposure ranging from 10 to $20 \mathrm{mSv}$ per WLM. Because of that, with respect to the preceding legislation, the 2013/59/EURATOM established a "Reference Level" of $300 \mathrm{~Bq} \mathrm{~m}^{-3}$ for annual average indoor radon concentrations (including both occupational and public exposures), overcoming the $500 \mathrm{~Bq} \mathrm{~m}^{-3}$ "Action Level" of the 1996/29/EURATOM (regarding occupational exposures only).

It should be considered that the 2013/59/EURATOM Directive advises Member States that National Reference Level for radon "shall not be set higher than $300 \mathrm{~Bq} \mathrm{~m} \mathrm{~m}^{-3}$ ", encouraging to choose lower values in situations involving long-term exposures to radon, i.e., workplaces and dwellings. Moreover, being the $300 \mathrm{~Bq} \mathrm{~m}^{-3}$ value conceived as a "reference level" instead of an "action level", radon exposures are subjected to the ALARA 
principle (As Low As Reasonably Achievable) and the annual average radon indoor concentration should be kept below $300 \mathrm{~Bq} \mathrm{~m}^{-3}$ as more as reasonably achievable.

With all these premises and the potential market related to radon issues and radiation protection aspects, the increasing number of new radon detectors developed in last years and service companies available for radon measurements can be understood. However, regardless any future legal implication, manufacturers or measurements providers can rely on the fact that radon is acknowledged as the second cause of lung cancer after cigarette smoking [6], [7]: in many cases it is a sufficient expedient to promote and diffuse their products to the public without any legal obligation.

The current and common tendency for manufacturers is to conceive "smart" devices even for radon measurements. e.g., Wi-Fi sensors with associated database and detectors which can be geo-localized allowing measurements linked to the specific features of the territory. Further, any member of the public could be able to measure indoor radon concentration by itself and receive notifications about high radon concentration with a message on his smart phone, etc.

A "smart" radon detector could be useful to people truly, as an alternative solution to traditional remedial action applied to "radon-rich" locations. Currently, in most Member States, when at a specific location the annual radon indoor concentration overcomes the "action level", remedial actions need to be applied to reduce such concentration. Typical actions are ventilation with external air, isolation of the building from foundations, "pressure traps" to intercept radon paths to the interior of the building, etc. Actions like these are usually applied continuously, regardless the effective indoor radon concentration. Here instead, a "smart" radon detector could be suitable to control, suggest, and optimize the remedial action operation, e.g., powering fans and active parts only when needed (only when people are there and concentrations are exceeded), or warning people at the place to open the window and dilute the internal air with external air. This way, radon concentrations would be controlled only when it is necessary, with relevant savings in costs and energy consumptions of buildings.

Such an hypothesis would suggest precise features for the "smart" radon detector to be applied: $i$ ) the sensor should be an active detector; ii) accuracy and precision of measurements in "following" radon evolution vs. time (day-night cycles) should be demonstrated; iii) responses should be in real time e.g., frequency of measurements could be hourly, or each quarter, depending on device's detection limits.

At the Radiation Protection Laboratory of the Sapienza University of Rome, Department of Basic and Applied Sciences for Engineering, a photodiode-based detector for alpha particles has been developed and properly characterized. All the features of the device and results about the experimental campaign carried out can be found in Gugliermetti et al. [8]. The detector is open-source, it is called "Alphaino", and it can be suitable for different applications involving alpha particles detection and spectroscopy, so including measurements of radon and its progeny. The paper herein discusses how the "Alphaino" detector has been suitably modified to build a radon progeny monitor, called "Radonino".

At the Radiation Protection Laboratory, an experimental campaign with the "Radonino" detector, the AlphaGUARD [12] radon monitor, and the Tracerlab BWLM-PLUS-2S [10] PAEC monitor, has been carried out. Synchronized and contemporary sets of measurements have been registered for an entire month. Part of the data acquired have been used for the initial calibration of the "Radonino" detector. With calibration achieved, data have been used to verify the detector operation and demonstrate its capability in "following" radon and progeny evolution vs. time, as for as professional and expensive instrumentation. 
This paper aims to show that reliable radon exposure measurements can be achieved even without expensive instrumentation, through a well-conceived detector. An effective and cheap radon monitor detector measuring directly the PAEC could be particularly suitable to provide continuous effective dose evaluation in radon-affected dwellings and workplaces, in order to warn people or initiate mitigation actions when truly needed.

\section{DETECTOR DEVELOPMENT}

The core of "Radonino" is the "Alphaino" detector discussed in Gugliermetti et al. [8]. Alphaino is an alpha-particle detector intended for applications where low cost and small dimension are key factors. It can be used both as an alpha particles counter, and for alpha spectroscopy. It can also be connected both to traditional nuclear spectroscopy electronics, and to cheap prototyping boards, e.g. Arduino [15]. The detector has been tested for low-rate alpha-particle counting and spectroscopy, demonstrating a maximum achievable count rate of $4 \cdot 10^{3} \mathrm{~s}^{-1}$, with an energy resolution corresponding to a full width at half maximum of 160 $\mathrm{keV}$ over the entire energy range of measured alpha, namely $4 \div 6.5 \mathrm{MeV}$, the intrinsic efficiency being $100 \%$. The detector is open-source: everyone can download the schematics, build the sensor and contribute to further development through the GITHUB repository for the project, https://github.com/bemxgm/Radon-Monitor.

The application of "Alphaino" to radon indoor concentration measurements comes from the fact that risks to people's health is given by the radon progeny, and not by radon gas itself. Detectors measuring radon $\left({ }^{222} \mathrm{Rn}\right.$ or $\left.{ }^{220} \mathrm{Rn}\right)$ gas directly can be fairly complex, and very expensive, due to the fact that radon isotopes are dispersed in air and radon is a noble gas, chemically inert almost. Detecting ${ }^{222} \mathrm{Rn}$ (or ${ }^{220} \mathrm{Rn}$ ) decays means to "observe" a certain volume of the air to be monitored with the experimental capability to detect the decay event in such control volume: ion chambers or scintillation cells are used, typically. Professional instrumentation exploits this kind of sensitive element, usually.

Being ${ }^{222} \mathrm{Rn}$ (and the same ${ }^{220} \mathrm{Rn}$ ) the initiator of an equilibrium chain with its $\mathrm{Po}, \mathrm{Pb}$, and Bi daughters, i.e., the progeny, detectors can rely on progeny measurements to infer the father concentration-in-air. The technical realization of the equilibrium among radon and its progeny is a quite interesting possibility to measure radon. As a matter of fact, the detection of the progeny is quite easier with respect to the measurement of the radon itself. Indeed, ${ }^{222} \mathrm{Rn}$ daughters, ${ }^{218} \mathrm{Po},{ }^{214} \mathrm{~Pb},{ }^{214} \mathrm{Bi}$, and ${ }^{214} \mathrm{Po}$, are mainly produced by decay in form of positive ions [9]. Such atoms have a high probability to get attached among themselves and/or with dust particulate in air, forming "clusters" of different sizes [10], [11]. Such "clusters" are called "unattached" (with diameter ranging from 0.5 to $4 \mathrm{~nm}$ ) when free or attached to vapor or gas in traces, and "attached" (with diameter from 0.1 to $0.4 \mu \mathrm{m}$ ) when attached to aerosol. The advantage in measuring radon's daughters is that the progeny can be easily collected in front of a detector for the measurement. Collection can be achieved by mechanical methods, e.g., forcing air to pass through a filter placed in front of the detector, or with electrostatic precipitation toward the sensor.

The "Alphaino" detector in Gugliermetti et al. [8] has been arranged in a suitable configuration to realize the mechanical collection of radon progeny on a paper filter by means of an air flow forced to pass throughout. The detector, Fig. 1, is called "Radonino". The photodiode sensor in front of the filter is capable in detecting alpha particles emerging from the filter itself. ${ }^{218} \mathrm{Po},{ }^{214} \mathrm{~Pb},{ }^{214} \mathrm{Bi}$, and ${ }^{214} \mathrm{Po}$ are determined by the analysis of the alpha particles' energy spectrum collected for one hour. By knowing the flow rate processed by the sensor, the Potential Alpha Energy Concentration, PAEC $\left.(\mathrm{MeV} \mathrm{cm})^{-3}\right)$, is calculated. Such energy, in form of a concentration of alpha particles decays in air, determines the risk to human health when air is breathed in the respiratory tract. 


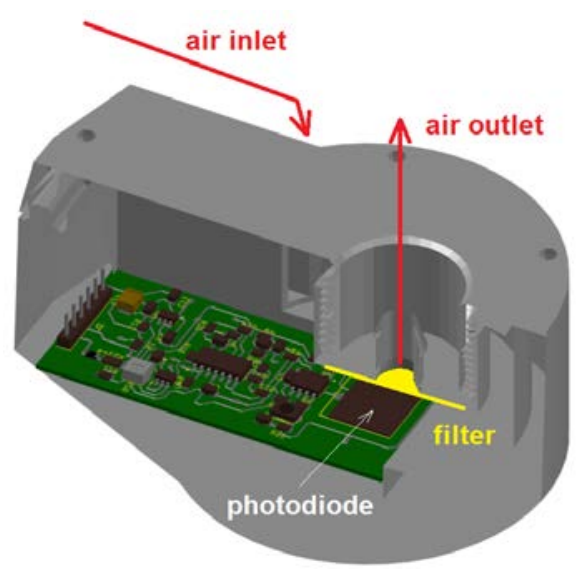

Figure 1: The "Radonino" detector. The chip is housed in a light-proof electromagnetic shielded ABS box sketched in gray color.

With the PAEC determined, it is possible to retrieve the corresponding radon concentration-in-air, being "action" or "reference" levels defined by legislation expressed in such units, or convert the PAEC in effective dose rate directly. Fig. 2 shows a comparison between the workflow with the "Radonino" detector here proposed and the classical workflow applied to professional radon detectors such as Bertin Instruments AlphaGUARD [12]. For the equilibrium factor, F, defined as the ratio between equivalent equilibrium concentration (EEC, i.e., the concentration corresponding to the PAEC) and the corresponding radon concentration-in-air, a nominal value of $\mathrm{F}=0.4$ is assumed for indoor locations, conventionally. As to convert PAEC in effective dose, is assumed the effective dose coefficient per unit exposure by ICRP 115 [13], i.e. $0.465 \mu \mathrm{Sv} \mathrm{cm}^{3} \mathrm{~h}^{-1} \mathrm{MeV}^{-1}$.

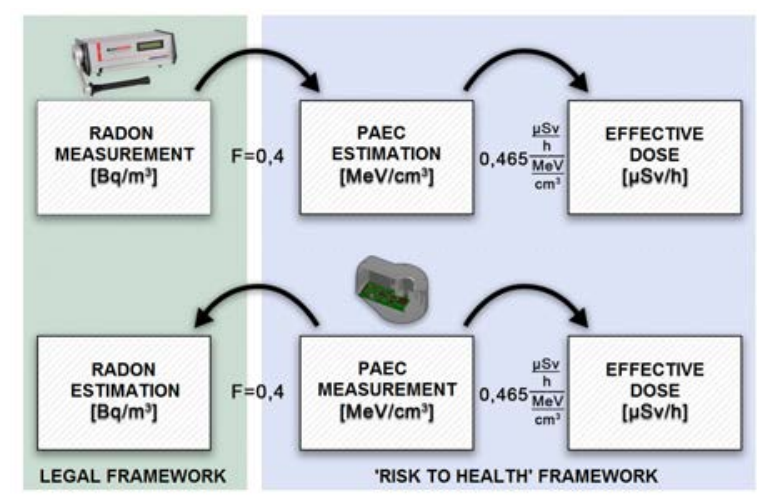

Figure 2: Workflow with professional radon monitors and with the "Radonino" detector here proposed.

The adjustment of "Alphaino" towards "Radonino" for realizing PAEC measurements asked for a new design of the casing with respect to Gugliermetti et al. [8]. The sensor is 
based on photodiode which is sensitive to light intrinsically, and a light-tight environment is needed for correct operations. For reducing the electronic noise, the casing has been covered with a conductive paint electrically connected to the ground, as to realize an electromagnetic shielding. The case has been produced by means of a 3D printer, and material selected is ABS, Acrilonitrile Butadiene Styrene, due to its excellent mechanical features. It allows the suction of air from the side and the outlet upright with respect to the photodiode sensitive element as depicted in Fig. 1. Prior to be expelled, the air is forced to pass throughout a suitable millipore paper filter, permitting the "dust" carrying the radon progeny to be settled, for alpha-particle detection.

The air flow is obtained by means of a volumetric micropump connected to the outlet of the detector. The flow rate realized corresponds to $0.3 \mathrm{~L} / \mathrm{s}$. Alpha particles energy spectra are recorded by means of a multi-channel analyzer ORTEC EASY-MCA-2k, and spectra are acquired with a frequency of one hour.

\section{EXPERIMENTALS}

The first test carried out was intended to demonstrate the feasibility of the project, i.e., verifying the effective capability of "Radonino" in detecting alpha particles from the radon progeny settled on the millipore paper filter.

Fig. 3 shows the first alpha energy spectrum measured with the "Radonino" detector, the acquisition lasting $24 \mathrm{~h}$ as to obtain statistically significant data to be compared with the professional detector Tracerlab BWLM-PLUS-2S. Peaks in the spectrum are due, in the order, to ${ }^{218} \mathrm{Po},{ }^{214} \mathrm{Po}$, and ${ }^{212} \mathrm{Po}$, the last one belonging to the ${ }^{220} \mathrm{Rn}$ decay chain.

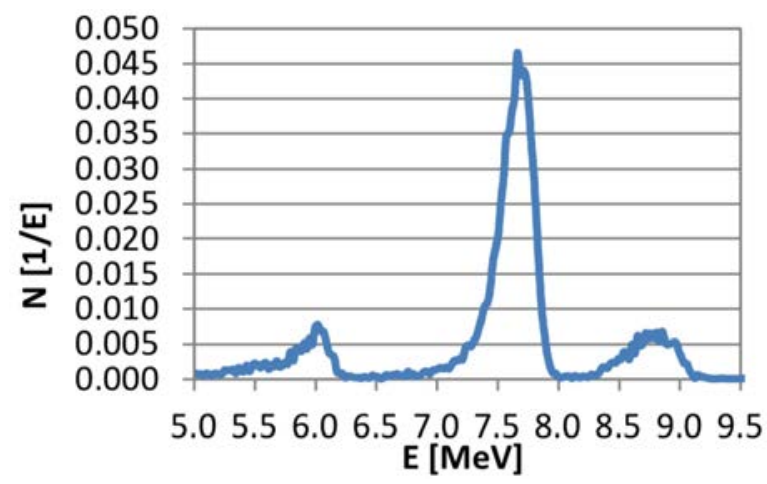

Figure 3: Alphaino recorded alpha spectrum of the progeny of ${ }^{220} \mathrm{Rn}-{ }^{222} \mathrm{Rn}$. Peaks in the spectrum are due to ${ }^{218} \mathrm{Po}(6 \mathrm{MeV}),{ }^{214} \mathrm{Po}(7.7 \mathrm{MeV}),{ }^{212} \mathrm{Po}(8.8 \mathrm{MeV})$.

The following experimental campaign was carried out for a quantitative characterization of "Radonino" aimed to calculating the efficiency of the system in detecting alpha particles vs. other working parameter selected, e.g. the air flow-rate. The calibration has been achieved by means of the Tracerlab BWLM-PLUS-2S. Alpha energy spectra, acquired with a frequency corresponding to 1 hour with both detectors synchronized in time, have been compared and the overall efficiency of "Radonino" in detecting alpha from the millipore filter was calculated.

Once ${ }^{218} \mathrm{Po}$ and ${ }^{214} \mathrm{Po}$ concentration-in-air are determined with the calibrated "Radonino", contributions to PAEC from ${ }^{214} \mathrm{~Pb}$ and ${ }^{214} \mathrm{Bi}$ can be inferred by equilibrium considerations. The PAEC value $\left[\mathrm{MeV} / \mathrm{cm}^{3}\right]$ vs. time is so determined for the following evaluations. 
Three different experimental campaigns have been realized at the Radiation Protection Laboratory, involving the contemporary and synchronized use of:

- AlphaGUARD PQ2000 to measure indoor ${ }^{222} \mathrm{Rn}$ concentrations, one value per hour;

- Tracerlab BWLM-PLUS-2S to measure PAEC, one value per hour;

- The "Radonino" detector to measure PAEC, one value per hour.

The three experimental campaigns differ in duration and boundary conditions (open or closed windows, day-night cycle, build-up effect due to closed windows in the weekend, weather, etc.). Those variations were intended to analyze the real spectrum of situations for indoor conditions, as to include the dynamic evolution of radon at the building in dose evaluations and results. In particular:

- Experimental Campaign \#1: working week, 88 hours, normal use for the analyzed room; weather: cloudy and rainy (low air pressure);

- Experimental Campaign \#2: two working days, 45 hours, normal use for the analyzed room; weather: sunny (high air pressure);

- Experimental Campaign \#3: weekend, 33 hours, room and building unused; weather: sunny (high air pressure).

As to discuss results, the dose approach in Fig. 2 (the "Risk to Health Framework") has been chosen to compare data. AlphaGUARD radon indoor concentrations measured values were converted in effective dose rate assuming $\mathrm{F}=0.4$ and $0.465 \mu \mathrm{Sv} \mathrm{cm} \mathrm{h}^{3} \mathrm{heV}^{-1}$. The same way, the $300 \mathrm{~Bq} \mathrm{~m}^{-3}$ "Reference Level" from 2013/59/EURATOM has been converted in the corresponding effective dose rate. The PAECs measured by Tracerlab and "Radonino" were converted to effective dose through the coefficient $0.465 \mu \mathrm{Sv} \mathrm{cm}{ }^{3} \mathrm{~h}^{-1} \mathrm{MeV}^{-1}$. Figs 4, 5 and 6 shows the three sets of data.

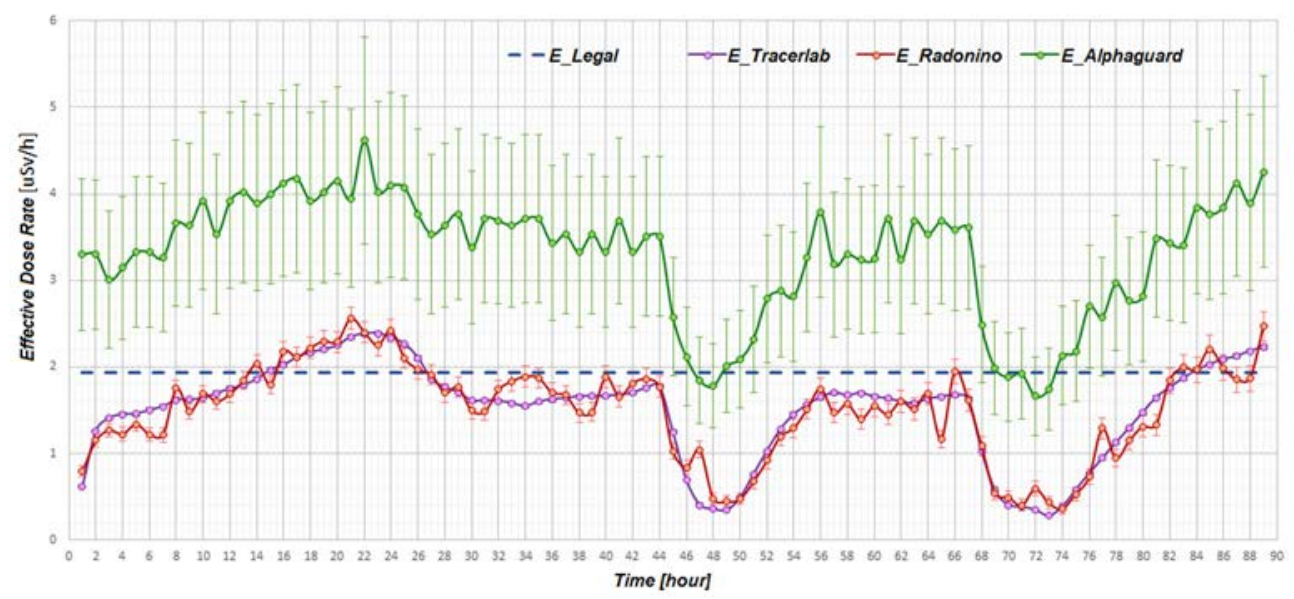

Figure 4: Results of the first experimental survey: comparison between effective dose rate computed from Radonino, Tracerlab BWLM-PLUS-2S, and AlphaGUARD measurements. 2013/59/EURATOM "Reference Level" converted to effective dose rate is provided as "E_Legal". This first set of measurements was performed during a whole week in a room regularly occupied by workers. The room is ventilated through windows manually operated according to occupants' arbitrary choices. $\mathrm{F}=0.18 \pm 24.3 \%, \mathrm{fp}=0.13 \pm 15.1 \%$. 


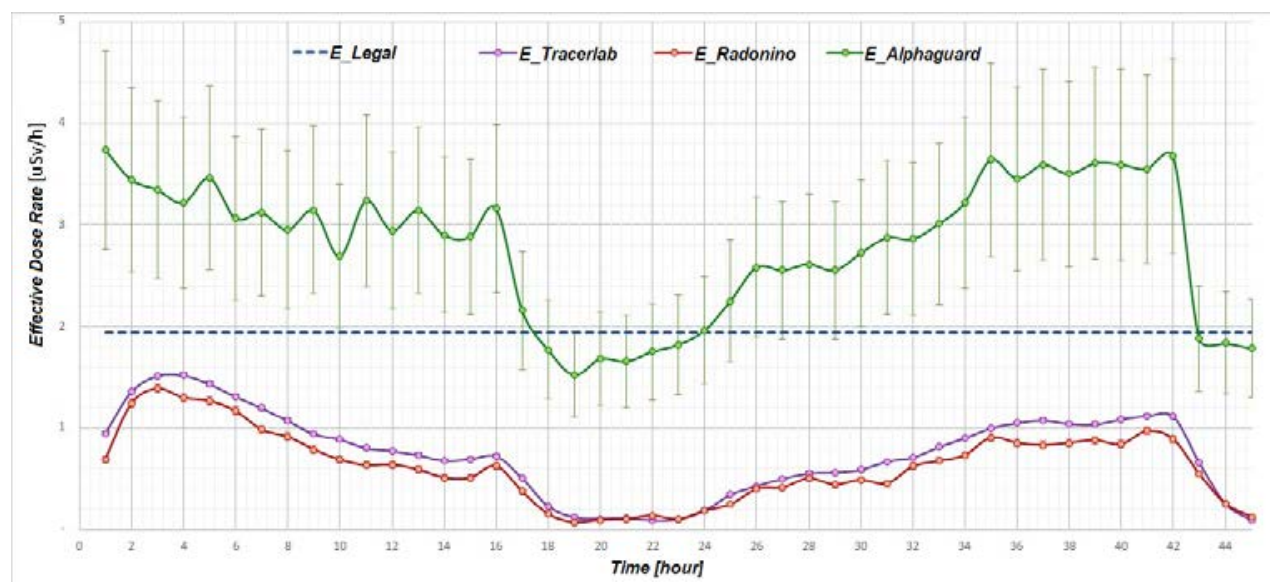

Figure 5: Results of the second experimental survey: comparison between effective dose rate computed from Radonino, Tracerlab BWLM-PLUS-2S, and AlphaGUARD measurements. 2013/59/EURATOM "Reference Level" converted to effective dose rate is provided as "E_Legal". This second set of measurements was performed in the same room as the first survey, but during the weekend. The room was closed and unattended during the whole period. $\mathrm{F}=0.21 \pm 29.8 \%$, $\mathrm{f}_{\mathrm{p}}=0.11 \pm 33.0 \%$.

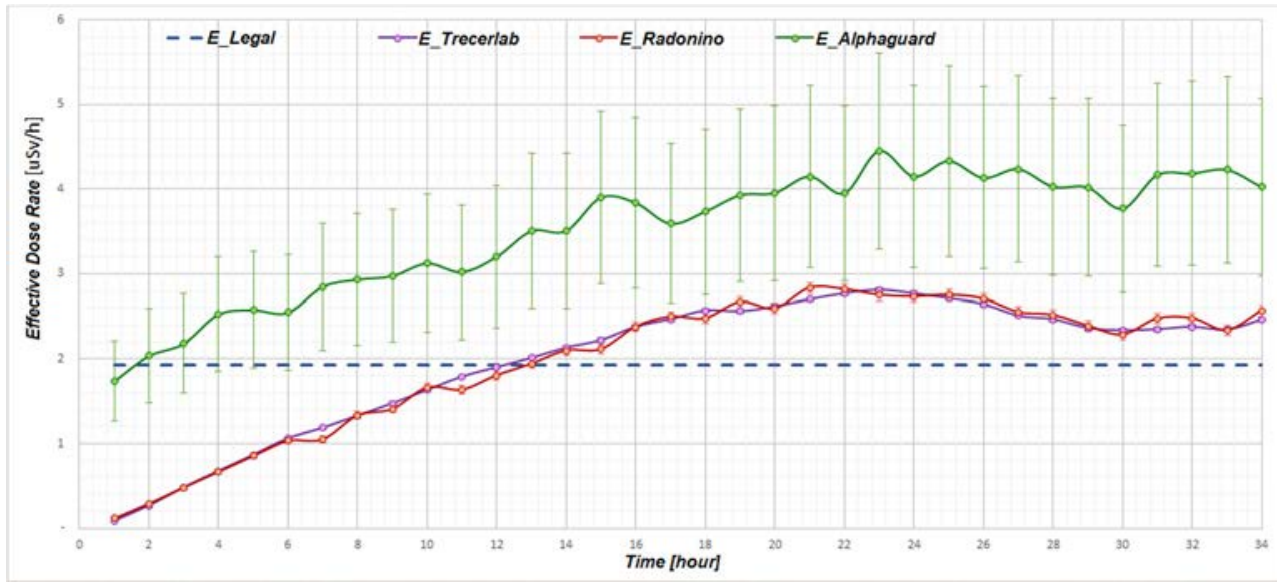

Figure 6: Results of the third experimental campaign: comparison between effective dose rate computed from Radonino, Tracerlab BWLM-PLUS-2S, and AlphaGUARD measurements. 2013/59/EURATOM "Reference Level" converted to effective dose rate is provided as "E_Legal". This third set of measurements was performed in the same room as the first two surveys. The observed period covered the transition from Sunday (weekend) to Monday (first day of working week). Due to the usual occupancy patterns, these hours are generally characterized by strong changes in all the climatic parameters (i.e., T, P, RH), as well as in Rn concentration, $F$, and $f_{p} . F=0.10 \pm 43.8 \%, f_{p}=0.29 \pm 22.0 \%$. 
Some considerations follow:

- The calibrated "Radonino" is capable in determining PAEC values vs. time, as for the professional detector Tracerlab BWLM-PLUS-2S. The matching in PAEC means matching in effective dose rates values vs. time, as can be seen from Fig. 4.

- Indoor radon concentrations measured with AlphaGUARD PQ2000 (converted in $\mathrm{PAEC}$ by $\mathrm{F}=0.4$ and effective dose rate later as happen for previous instruments) return overestimations of the effective dose rate due to the selected value $\mathrm{F}=0.4$, assumed for indoor environments conventionally.

- At the room analyzed, the equilibrium factor $F$ has been measured with Tracerlab BWLM-PLUS-2S vs. time. Experimental values show that $\mathrm{F}=0.4$ can be assumed as a conservative assumption in this case, being the true value measured lower.

- Evaluate radon exposure starting from PAEC determination, or indoor radon concentration determination (Fig. 2), may lead to different results, as measurements and following calculations in Fig. 4 demonstrate; e.g. the 1st and 2nd experimental campaigns in Fig. 4 show that the exposure evaluation starting from radon measurement overcomes the legal 2013/59/EURATOM "threshold" (being F=0.4 an overestimation), while true values remain under such legal "threshold".

\section{CONCLUSIONS AND FUTURE PERSPECTIVES}

The paper discussed the application of the Alphaino sensor developed by the authors in Gugliermetti et al. [8] to radon measurements and the following effective dose evaluations.

The detector, named "Radonino" measured the Potential Alpha Energy Concentration in air, PAEC $\left[\mathrm{MeV} \mathrm{cm}{ }^{-3}\right]$, vs. time. It has been calibrated by means of professional instrumentation, Tracerlab BWLM-PLUS-2S and AlphaGUARD PQ2000.

Three experimental campaigns (variable in duration; boundary conditions, i.e., open or closed windows, day-night cycle, build-up effect due to closed windows in the weekend, weather; etc.) have been carried out with all instruments synchronized and with a 1-hour time resolution. Results showed that the inexpensive "Radonino" is capable in measuring PAEC vs. time as for the professional Tracerlab BWLM-PLUS-2S. PAEC values were converted to the corresponding radon concentration $\left[\mathrm{Bq} \mathrm{m}^{-3}\right]$ by means of the equilibrium factor $\mathrm{F}$, or to effective dose rate directly.

Experimental measurements here discussed demonstrate that "Radonino" could be suitable for future developments in terms of an inexpensive radon monitor with professional performances. Future works will include the coupling with inexpensive electronic board targeted for project developing, such as Arduino, as to register alpha energy spectra, to control the pump, to compute the detector response, and to start mitigating actions e.g. initiate mechanical ventilation, or warn the user to open the window and dilute indoor concentrations with external air.

\section{REFERENCES}

[1] EU Council, Council directive 2013/59/Euratom. Official Journal of the European Union, 17(1), 2014.

[2] NRC, Health effects of exposure to Radon. BEIR VI Report, National Academy Press: Washington, DC, 1999.

[3] Tomasek, L., Rogel, A. \& Tirmarche, M., Lung cancer in French and Czech uranium miners risk at low exposure rates and modifying effects of time since exposure and age at exposure. Radiat. Res., 169, pp. 125-137, 2008. 
[4] Lecomte, J.-F. et al., Radiological protection against radon exposure. Ann. ICRP, 43(3), 2014.

[5] International Commission on Radiological Protection (ICRP), Human Respiratory Tract Model for Radiological Protection, ICRP Publication 66, 1994.

[6] United Nations Scientific Committee on the Effects of Atomic Radiation, Sources and Effects of Ionizing Radiation, New York, 2008.

[7] World Health Organization, Indoor radon: A public health perspective. International Journal of Environmental Studies, 67(1), 2009.

[8] Gugliermetti, L., Lepore, L., Remetti, R. \& Colarieti Tosti, M., Alpha spectrometry with the inexpensive open-source detector Alphaino. Nuclear Instruments and Methods in Physics Research Section A: Accelerators, Spectrometers, Detectors and Associated Equipment, 928, pp. 13-19, 2019.

[9] National Council on Radiation Protection and Measurements, Measurement of radon and radon daughters in air. Report no. 97, 1988.

[10] Porstendörfer, J., Properties and behaviour of radon and thoron and their decay products in the air. J. Aerosol Sci., 25(2), pp. 219-263, 1993.

[11] Porstendörfer, J., Pagelkopf, P. \& Gründel, M., Fraction of the positive 218-Po and 214-Pb clusters in indoor air. Radiation Protection Dosimetry, 3, pp. 342-351, 2005.

[12] Bertin Instruments. AlphaGUARD: Radon Monitor. www.bertin-instruments.com/ product/radon-professional-monitoring/radon-alphaguard/. Accessed on: 9 May. 2019.

[13] Tirmarche, M., Harrison, J., Laurier, D. Paquet, F., Blanchardon, E. \& Marsh, J., Lung cancer risk from radon and progeny and statement on radon. Ann. ICRP, 40(1), 2010.

[14] Tracerlab. GmbH, 2018. www.tracerlab.com/cataloge/index.htm?http://www.tracer lab.com/cataloge/documents/wlmdesc.htm.

[15] Arduino, Arduino UNO. www.arduino.cc/en/Guide/ArduinoUno. 